\title{
Connective tissue remodelling is differently modulated by tocilizumab versus methotrexate monotherapy in patients with early rheumatoid arthritis: the AMBITION study
}

Patryk J. Drobinski ${ }^{1,2^{*}}$, Anne C. Bay-Jensen ${ }^{1}$, Morten A. Karsdal ${ }^{3}$, Samra Sardar ${ }^{1}$ and Anne S. Siebuhr ${ }^{1}$

\begin{abstract}
Objective: Associations between rheumatoid arthritis (RA) and effect of treatment at the tissue levels are poorly understood. We investigated the scope of released extracellular matrix (ECM) metabolites as a consequence of tissue remodelling in patients treated with methotrexate (MTX) and tocilizumab (TCZ) compared to placebo.

Methods: Tissue metabolites from 387 RA patients treated with either TCZ ( $8 \mathrm{mg} / \mathrm{kg}$ ) or MTX monotherapy $(7.5-20 \mathrm{mg} / \mathrm{kg})$ were measured at baseline and 8 weeks sera by validated ELISA assays. The levels of collagen biomarkers (C1M, C2M, C3M and C4M) together with C-reactive protein (CRP) and CRP metabolite (CRPM) were investigated. Baseline levels of biomarkers have been compared with 72 age- and gender-matched healthy controls. Comparison between treatment and response groups were done by ANCOVA, Spearman's correlation and logistic regression adjusted for age, gender, BMI and disease duration.

Results: C1M and C3M were significantly $(P<0.05)$ inhibited by TCZ and C3M by MTX $(P<0.01)$ compared to placebo. C1M and C3M inhibition with TCZ was respectively $23 \%$ and $16 \%$ greater than that of MTX $(P<0.01$ and $P<0.0001)$. C4M was inhibited by TCZ and MTX, but the effect of TCZ was 22\% greater than MTX $(P<0.0001)$. TCZ and MTX had minimal effect on C2M levels. MTX had no effect on CRP and CRPM, whereas TCZ reduced their levels to 69\% and 27\% from baseline. Investigated biomarkers revealed a significant $(P<0.05)$ difference in biomarker profiles of MTX ACR50 treatment responders and non-responders. Change to week 8 in levels of C3M, C4M, CRP and CRPM in MTX patients correlated significantly ( $r$ ho $=0.41$ to $0.18, P<0.0001$ to 0.039 ) with change in disease activity (DAS28) at weeks 8,16 and 24, whereas only CRP in TCZ patients (rho $=0.32$ to $0.21, P<0.0001$ to 0.01 ).

\footnotetext{
* Correspondence: pad@nordicbio.com

'ImmunoScience, Nordic Bioscience, Herlev Hovedgade 207, DK-2730 Herlev, Denmark

${ }^{2}$ Department of Biomedical Sciences, University of Copenhagen, Blegdamsvej 3, 2200 Copenhagen N, Denmark

Full list of author information is available at the end of the article
}

C C The Author(s). 2020 Open Access This article is licensed under a Creative Commons Attribution 4.0 International License, which permits use, sharing, adaptation, distribution and reproduction in any medium or format, as long as you give appropriate credit to the original author(s) and the source, provide a link to the Creative Commons licence, and indicate if changes were made. The images or other third party material in this article are included in the article's Creative Commons licence, unless indicated otherwise in a credit line to the material. If material is not included in the article's Creative Commons licence and your intended use is not permitted by statutory regulation or exceeds the permitted use, you will need to obtain permission directly from the copyright holder. To view a copy of this licence, visit http://creativecommons.org/licenses/by/4.0/ The Creative Commons Public Domain Dedication waiver (http://creativecommons.org/publicdomain/zero/1.0/) applies to the data made available in this article, unless otherwise stated in a credit line to the data. 


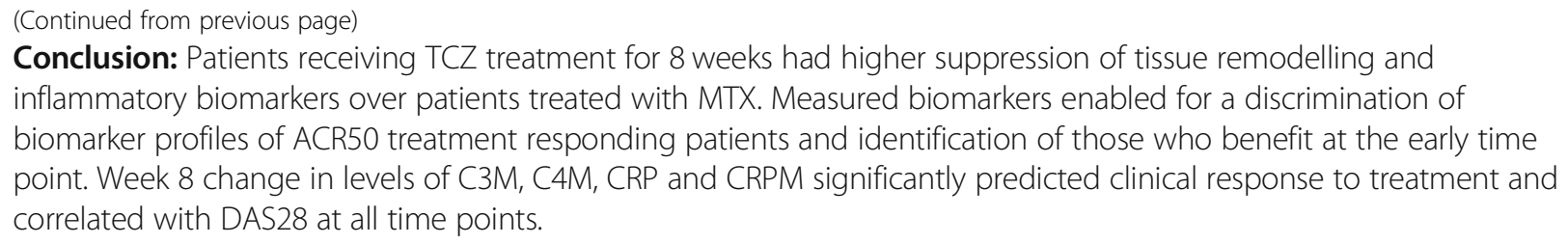

Trial registration: ClinicalTrials.gov, NCT00109408. Date of registration: July 2005. Name of the registry: A Study to Assess the Safety and Efficacy of Tocilizumab in Patients with Active Rheumatoid Arthritis.

Keywords: Tocilizumab (TCZ), Methotrexate (MTX), Rheumatoid arthritis (RA), Biomarkers, Tissue remodelling, Extracellular matrix (ECM)

\section{Introduction}

Rheumatoid arthritis (RA) is a complex autoimmune, inflammatory disease, which primarily affects synovial joints and causes local damage along with systemic manifestations $[1,2]$. It is prevalent in $0.5-1 \%$ of the world's population. The disease has a progressive course leading to permanent disability and is therefore associated with extensive personal, social and economic impacts. Despite decades of research into this disease, the aetiology of RA is still not completely understood but is known to be dependent on both genetic susceptibility and environmental factors [3].

The introduction of disease-modifying anti-rheumatic drugs (DMARDs) such as methotrexate (MTX) has substantially changed the prognosis and improved everyday life of RA patients. MTX relieve disease symptoms and remains the most commonly used disease-modifying antirheumatic drug $[4,5]$. However, therapies involving MTX often have inadequate response or intolerance, which calls for alternative treatments such as biological agents [6]. One of them is tocilizumab (TCZ), a humanised anti-IL-6 receptor monoclonal antibody, which acts by blocking IL6 signalling [7-9]. Elevated levels of IL-6 in synovial fluid and serum correlate with disease activity in patients with RA $[10,11]$. Inhibition of the IL-6 signalling by TCZ showed a protective effect on bone erosions by decreasing their volume in RA patients and significant reduction of swollen and tender joint counts with concurrent improvement in radiographic progression $[9,12]$.

Key histological features of RA affected joints include hyperplasia and cellular infiltration of the synovium and systemic inflammation [13]. The inflammation leads to accelerated remodelling of extracellular matrix (ECM) that can progress to bone erosion and cartilage destruction [14, 15]. Destruction of connective tissue, especially the ECM of cartilage, bone and soft tissues of the joint, is a hallmark of RA pathology [16].

Proteolytic enzymes expressed locally in the pathologically affected tissue, such as the matrix metalloproteinases (MMPs) and aggrecanases, have a role in the remodelling of the ECM.
In healthy connective tissues, physiologic expression of these enzymes is low, but increases considerably during the inflammation process in RA thereby leading to excessive ECM remodelling [17]. We have previously shown that MMP-3 was highly elevated in RA and treatment with TCZ significantly decreased MMP3 levels [18]. The consequence of MMPs upregulation is heightened ECM remodelling with the release of a range of protein-specific degradation products (neo-epitopes) to circulation that can be used as biomarkers of tissue remodelling in joint diseases [19]. Former findings have shown that upregulated levels of MMP-degraded type I, II, III and IV collagen (C1M, C2M, C3M and C4M, respectively) correlated with disease activity in RA patients and treatment with TCZ+MTX significantly suppressed the biomarkers dose-dependently [20-23]. C1M was shown not only to be associated with disease activity, but a useful tool for identification of RA patients with fast structural progression [22]. Gudmann et al. have shown that $\mathrm{C} 4 \mathrm{M}$ together with $\mathrm{C} 1 \mathrm{M}$ and $\mathrm{C} 3 \mathrm{M}$ are biomarkers reflecting structural joint degradation in RA and their baseline elevated levels significantly correlates with disease activity score of 28 joints (DAS28) [23].

Another type of biomarkers is biomarkers that indicate an ongoing inflammatory process. C-reactive protein (CRP), a biomarker of systemic inflammation, is produced and released from the liver as an acute reactant during inflammation, whereas CRPM, an MMPdegraded fragment of CRP, is released from the inflamed tissue. CRP levels have shown predict clinical outcomes from treatment with TCZ in RA and many other inflammatory diseases [24]. It was reported that levels of CRPM correlate with RA activity at different disease stages and significantly downregulate together with collagen biomarkers after TCZ+MTX treatment $[21,25,26]$. Serological biomarkers of tissue remodelling and inflammation may give insight into treatment effect at the tissue level of different interventions giving an alternative approach to currently used methods such as symptomatic changes assessed by ACR-response or DAS28. In our study, we investigated if tissue remodelling was differently modulated 
by TCZ and MTX monotherapies in RA patients by measuring tissue remodelling biomarkers.

\section{Materials and methods Patients and study design}

The AMBITION study (NCT00109408) was a 24-week phase 3 trial, double-blind, double-dummy, parallelgroup, including 673 biological naive patients, randomised to either tocilizumab (TCZ) monotherapy $8 \mathrm{mg} /$ $\mathrm{kg}$ intravenously every 4 weeks or methotrexate (MTX) monotherapy oral capsules every week (initial dose 7.5 $\mathrm{mg}$ and titrated to $15 \mathrm{mg}$ at week 4 and to $20 \mathrm{mg}$ at week 8) [27]. Placebo ( $\mathrm{PBO})$ patients received tocilizumab 8 $\mathrm{mg} / \mathrm{kg}$ from week 8 until the end of the study. The current biomarker sub-study of AMBITION involved baseline and week 8 serum samples from a total number of 387 adult patients ( $>18$ years) with moderate to severe active RA (Fig. 1). The AMBITION study was approved by the ethics committee at each participating institution (in USA, Canada and Israel) and was conducted in accordance with the principles of good clinical practice and according to the Declaration of Helsinki. All patients included provided written, informed consent before inclusion in the study. Serum from 72 healthy controls included in the study was obtained from the Discovery Life Sciences, Inc., vendor with compliance to the Ethics Committee recommendations and all regulations, guidelines and best practices that meet or exceed the US and international regulatory requirements.

\section{Biochemical marker assays}

Serum levels of type I [28], II [29], III [30] and IV [31] collagen degradation biomarkers together with CRP and its metabolite CRPM [25] were measured in serum by technically validated ELISAs developed by Nordic Bioscience (Herlev, Denmark). Biomarkers were measured according to manufacture instructions. A brief description of the protocol follows: Streptavidin-coated 96-well plates were coated with respective biotinylated antigens for 30 min at $20^{\circ} \mathrm{C}$ and subsequently washed 5 times in washing buffer. Appropriate controls, standard and serum samples were added with subsequent addition of peroxidase conjugated antibody against the respective epitope.

The mixture was incubated depending of an assay for $1 \mathrm{~h}$ at 20C (C3M, C4M, CRPM) or $20 \mathrm{~h}$ (reC1M, C2M) at $4{ }^{\circ} \mathrm{C}$. Plates were subsequently washed 5 times with washing buffer. TMB substrate $\left(3,3^{\prime}, 5,5^{\prime}\right.$-tetramethylbenzidine) was added and incubated for $15 \mathrm{~min}$ at $20^{\circ} \mathrm{C}$ and the reaction was stopped with $0.18 \mathrm{M}$ sulphuric acid. A SpectraMax Microplate Reader (Molecular Devices Corporation, Sunnyvale, CA, USA) was used to read the absorbance at $450 \mathrm{~nm}$ with reference set to $650 \mathrm{~nm}$. The concentrations were calculated by using a 4-parametric curve fit model. Biomarker results were approved under fulfilling acceptance requirements of an analytical run. This comprised the acceptance of three main criteria. Firstly, acceptance of the standard curve with all standards points resulting in $<10 \%$ coefficient of variance (CV) and $<15 \%$ of relative error (\%RE) within the

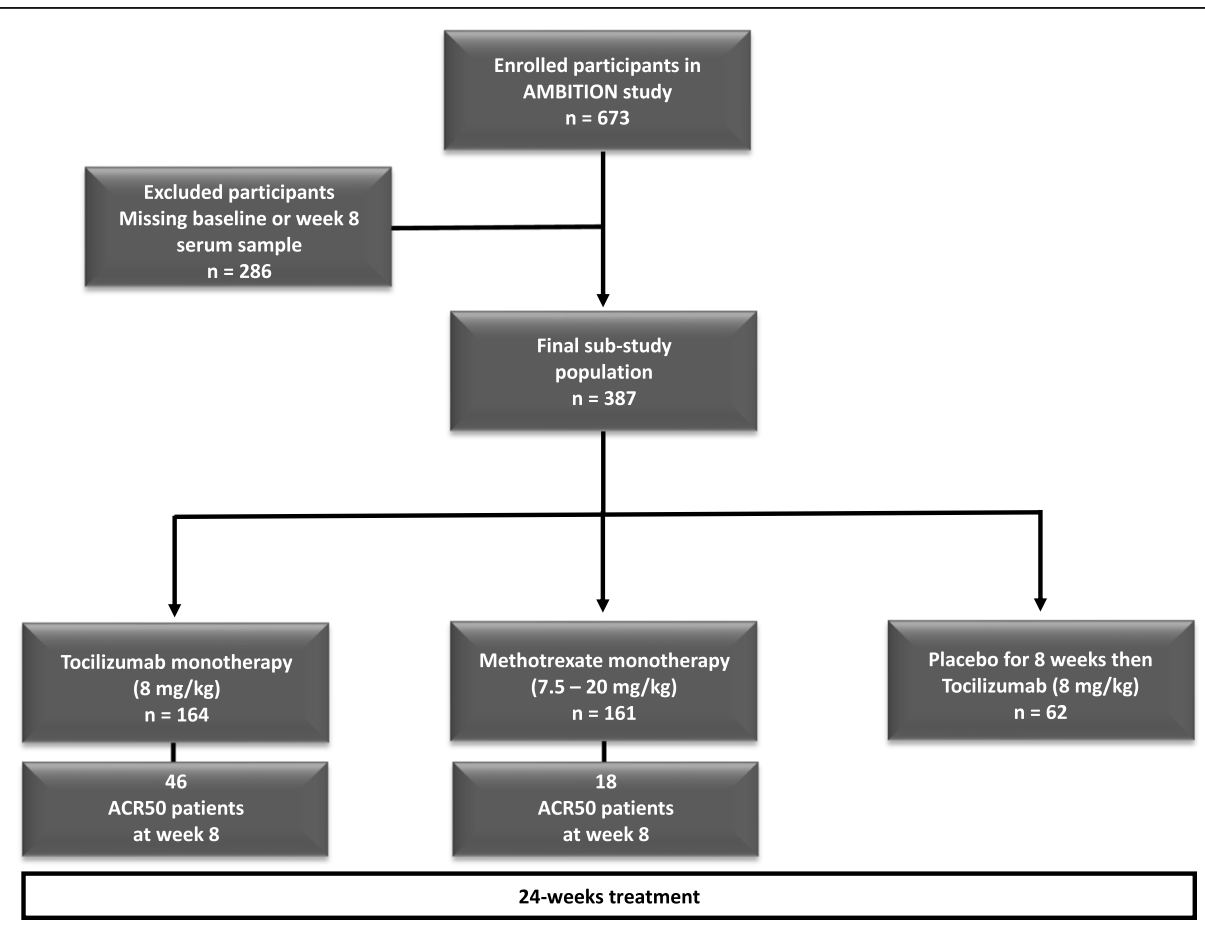

Fig. 1 Patient randomisation into three different treatment arms in the AMBITION study 
analytical measuring range. The exception from that criteria was that one standard point could be masked if $\mathrm{CV}>$ $10 \%$ or if $\% R E>15 \%$. Secondly, acceptance of the quality controls with all resulting in $<15 \% \mathrm{CV}$ for all QC specimens and within the target range of mean $\pm 20 \%$, with the exception that one QC specimen was allowed to have CV $>15 \%$. Lastly, acceptance of sample measurement for samples within the measurement range and $<15 \% \mathrm{CV}$. Samples with a CV $>15 \%$ were re-analysed.

\section{Statistical analyses}

The distribution of patient's baseline parameters among different treatment groups was assessed by one-way ANOVA $t$ test and presented in Table 1. Biomarker values that were above (ULOD) detection range were remeasured in dilution or excluded from the further analysis. The lower detection limit (LLOD) values were handled by setting the detection limit cutoff for confident determination of sample concentration and statistical differentiation from a blank at a 99\% confidence level. Change in biomarker levels at week 8 in relation to baseline was tested by paired $t$ test for each treatment (Fig. 2). Comparison between treatment groups (TCZ, MTX and PBO) was examined by
ANCOVA at baseline and week 8, adjusted for age, gender, BMI and disease duration.

Correlation between the change in biomarker levels from baseline to week 8 and disease activity (DAS28) at weeks 8, 16 and 24 was assessed with Spearman's correlation (rho) (Table 4). The normality of data distribution was assessed by Shapiro-Wilk test and significance between groups was assessed by Mann-Whitney nonparametric test. Data were presented as a mean with either 95\% confidence interval (CI-95\%), standard error of mean (SEM) or standard deviation of mean (SD). Statistical significance in mean biomarker change between treatment groups at baseline and week 8 were assessed by unpaired ANOVA $t$ test. Significance was considered when $P$ values were ${ }^{*} P<0.05,{ }^{* *} P<0.01,{ }^{* * * *} P<0.001$, ***:* $P<0.0001$. All statistical analyses were performed by using MedCalc version 14.8.1 and Prism Graphpad version 8.1.2 for graphical preparation.

\section{Results}

\section{Baseline demographics}

This current sub-study of AMBITION had 387 patients divided into three treatment arms with similar distribution of age, BMI, gender and disease activity (Table 1). There were no significant differences in baseline

Table 1 Patient demographics and baseline characteristics of the AMBITION cohort, according to treatment group. Differences between treatment groups are presented by one-way ANOVA $t$ test

\begin{tabular}{|c|c|c|c|c|}
\hline Characteristics & Tocilizumab, $N=164$ & Methotrexate, $N=161$ & Placebo, $N=62$ & $P$ value \\
\hline Age (years), mean (SD) & $50.7(12.9)$ & $51.3(13.3)$ & $50.5(11.8)$ & ns \\
\hline Female, $n(\%)$ & $131(80)$ & $122(76)$ & $47(76)$ & ns \\
\hline Male, $n(\%)$ & $33(20)$ & $39(24)$ & $15(24)$ & ns \\
\hline DAS28, mean (SD) & $6.8(0.9)$ & $6.8(0.9)$ & $6.9(0.9)$ & ns \\
\hline BMI (SD) & $28.1(6.4)$ & $27.4(6.0)$ & $29.1(7.4)$ & ns \\
\hline TJC, mean (SD) & $32.6(14.8)$ & $32.1(14.1)$ & $35.4(16.3)$ & ns \\
\hline SJC, mean (SD) & $19.4(10.9)$ & 19.9 (10.6) & $23.5(12.2)$ & $P=0.04$ \\
\hline RADUR (SD) & $6.7(8.1)$ & $6.6(8.0)$ & $7.4(9.0)$ & ns \\
\hline CRP (mg/dL), mean (SD) & $3.2(3.6)$ & $3.1(3.5)$ & $2.5(2.7)$ & ns \\
\hline ESR $(\mathrm{mm} / \mathrm{h})$, mean (SD) & $46.9(26.1)$ & $47.9(24.1)$ & $48.2(22.4)$ & ns \\
\hline HAQ-DI, mean (SD) & $1.6(0.6)$ & $1.6(0.6)$ & $1.4(0.5)$ & ns \\
\hline Pain VAS $100 \mathrm{~mm}$, mean (SD) & $59.0(22.4)$ & $61.3(20.7)$ & $59.5(24.4)$ & ns \\
\hline Patient VAS $100 \mathrm{~mm}$, mean (SD) & $64.6(22.0)$ & $64.9(19.4)$ & $64.3(22.5)$ & ns \\
\hline Physician VAS 100 mm, mean (SD) & $63.5(16.1)$ & $64.6(16.3)$ & $69.1(17.9)$ & ns \\
\hline Baseline C1M (ng/mL), mean $(95 \% \mathrm{Cl})$ & $42.7(37.5-47.8)$ & $45.2(39.4-50.9)$ & $40.5(34.5-46.5)$ & ns \\
\hline Baseline C2M (ng/mL), mean $(95 \% \mathrm{Cl})$ & $0.4(0.4-0.5)$ & $0.4(0.4-0.5)$ & $0.3(0.3-0.4)$ & ns \\
\hline Baseline C3M (ng/mL), mean $(95 \% \mathrm{Cl})$ & $17.8(16.5-19.2)$ & $18.3(17.1-19.5)$ & $15.9(13.8-17.9)$ & ns \\
\hline Baseline C4M (ng/mL), mean $(95 \% \mathrm{Cl})$ & $53.9(49.3-58.4)$ & $51(47.2-54.8)$ & $42.3(36.5-48.1)$ & $P=0.02$ \\
\hline Baseline CRPM $(\mathrm{ng} / \mathrm{mL})$, mean $(95 \% \mathrm{Cl})$ & $21.9(19.6-24.2)$ & $20.8(18.9-22.8)$ & $18.5(15.0-21.9)$ & ns \\
\hline Baseline CRP $(\mathrm{ng} / \mathrm{mL})$, mean $(95 \% \mathrm{Cl})$ & $3.2(2.6-3.8)$ & $3.1(2.6-3.7)$ & $2.5(1.8-3.2)$ & ns \\
\hline
\end{tabular}




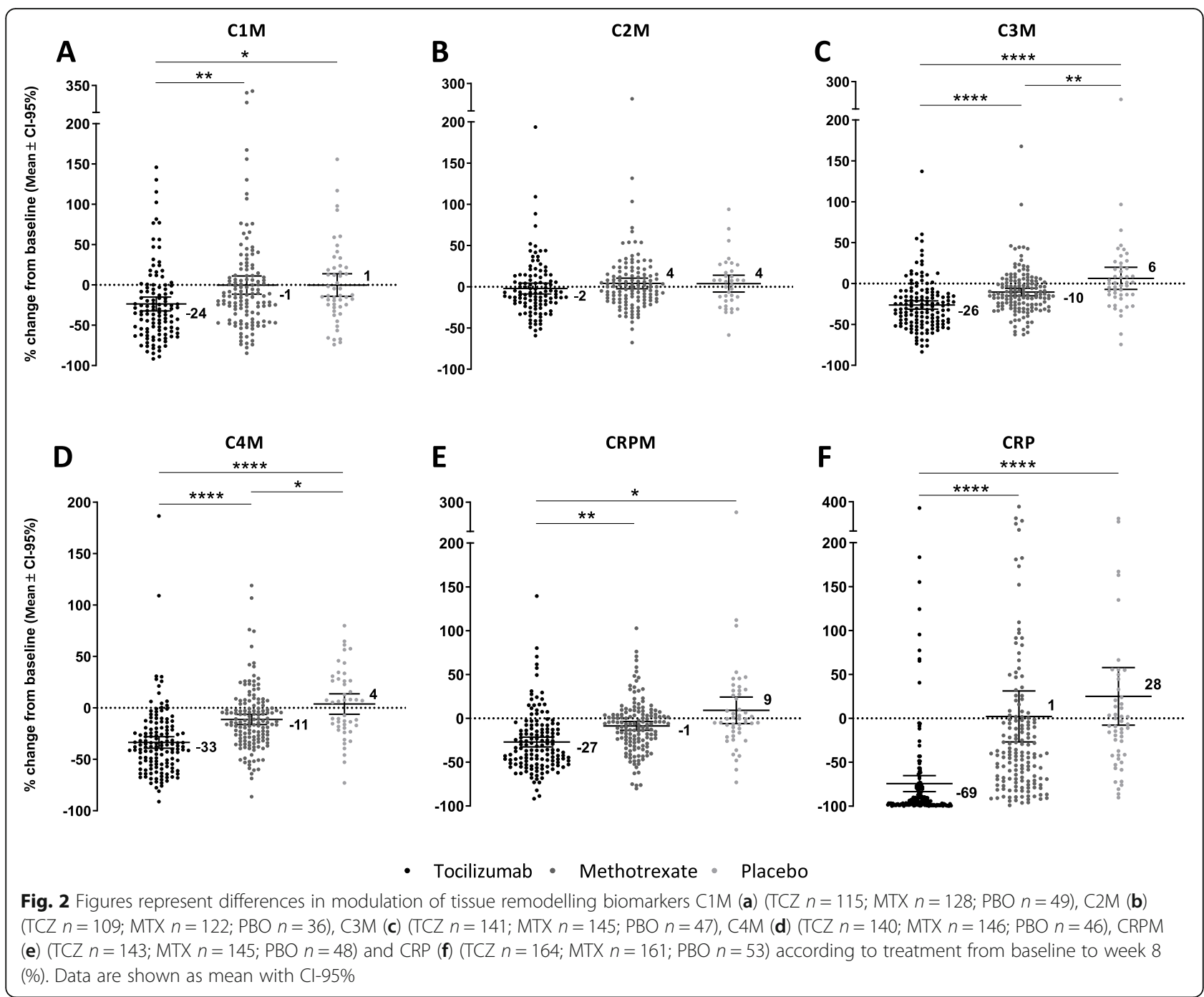

parameters between treatment groups except the number of swollen joint count (SJC) that was slightly higher in placebo patients $(P=0.04)$ and lower baseline C4M levels in placebo patients $(P=0.02)$. The same differences regarding clinical parameters were found in the complete AMBITION study (data not shown). All biomarkers except $\mathrm{C} 2 \mathrm{M}$ correlated significantly at baseline $(P<0.0001$ to $P<0.01$, Spearman's rho 0.15 to 0.31$)$ to the initial DAS28. The baseline levels of biomarkers in sub-study of AMBITION cohort were compared with healthy controls, involving 72 age- and gender-matched donors (Table 2). Comparison of these values was performed with ANCOVA adjusted for race differences and revealed significantly lower baseline levels of all biomarkers in healthy controls $(P<0.001)$.

\section{Biomarker modulation at week 8}

The week 8 levels of biomarkers associated with connective tissue remodelling, $\mathrm{C} 1 \mathrm{M}$ and $\mathrm{C} 3 \mathrm{M}$ were significantly downregulated by tocilizumab (TCZ) when compared to placebo $(P<0.05$ and $P<0.0001$, respectively) and $\mathrm{C} 3 \mathrm{M}$ also by methotrexate (MTX) compared to placebo $(P<0.01)$ (Fig. 2a, c). The inhibition of $\mathrm{C} 1 \mathrm{M}$ and $\mathrm{C} 3 \mathrm{M}$ with $\mathrm{TCZ}$ was, respectively, $23 \%$ and $16 \%$ greater than that of MTX $(P<0.01$ and $P<0001)$. The levels of the basement membrane remodelling biomarker C4M were likewise inhibited by both TCZ and MTX comparing to placebo $(P<$ 0.0001 and $P<0.05$, respectively) and the effect of TCZ was $22 \%$ greater than MTX $(P<0.0001)$ (Fig. $2 \mathrm{~d})$. In contrast, TCZ had minimal and MTX no effect on downregulation of the cartilage degradation biomarker $\mathrm{C} 2 \mathrm{M}$ when compared to placebo (Fig. 2b). Considering effect of MTX on inflammatory biomarkers modulation, both CRP and CRPM levels were downregulated at week 8 when compared to placebo, $27 \%$ and $10 \%$ decrease, respectively (Fig. 2e, f). TCZ showed greater than MTX inhibitory effect on CRP 
Table 2 Comparison of AMBITION cohort with healthy controls in patient demographics and baseline biomarker levels. Differences between cohorts are shown as ANCOVA values adjusted for race differences

\begin{tabular}{llll}
\hline Variables & AMBITION cohort RA patients $(\boldsymbol{N}=\mathbf{3 8 7})$ & Healthy controls $(\boldsymbol{N}=\mathbf{7 2})$ & Difference between cohorts \\
\hline Age (years), mean (SD) & $50.9(12.9)$ & $49.7(6.1)$ & $\mathrm{ns}$ \\
Female, $\boldsymbol{n}(\%)$ & $300(76)$ & $41(76)$ & $\mathrm{ns}$ \\
Race, no. white/black/other (\%) & $325(84) / 14(4) / 48(12)$ & $51(71) / 21(29) / 0(0)$ & $<0.0001$ \\
Baseline C1M (ng/mL), mean (SD) & $43.4(30.7)$ & $18.7(15.3)$ & $<0.001$ \\
Baseline C2M (ng/mL), mean (SD) & $0.38(0.2)$ & $0.3(0.16)$ & $<0.001$ \\
Baseline C3M (ng/mL), mean (SD) & $17.8(8.0)$ & $11.1(2.8)$ & $<0.001$ \\
Baseline C4M (ng/mL), mean (SD) & $50.9(24.9)$ & $21.5(6.1)$ & $<0.001$ \\
Baseline CRPM (ng/mL), mean (SD) & $20.9(12.9)$ & $7.5(2.1)$ & $<0.001$ \\
Baseline CRP $(\mathbf{n g} / \mathbf{m L})$, mean (SD) & $3.0(3.5)$ & - & \\
\hline
\end{tabular}

and CRPM resulting in $97 \%(P<0.0001)$ and $36 \%$ $(P<0.01)$ decrease comparing to placebo patients.

\section{Treatment-dependent difference in biomarker profiles of ACR50 responders and non-responders}

Patients of the TCZ and MTX groups who achieved ACR50 at week 8 were classified as treatment responders. Patients that did not meet ACR50 at week 8 were classified as treatment non-responders. The treatment with TCZ demonstrated to be better than MTX with a higher ACR50 response rate $(P=0.01)$ (Table 3$)$. Among the 164 patients that were treated with TCZ, 46 patients reached ACR50 at week 8 (28\%). In the MTX group, only 18 of the 161 patients reached ACR50 at week 8 (11\%).

$\mathrm{C} 1 \mathrm{M}$ among responders and non-responders in TCZ group did not show significant difference in the biomarker profiles at weeks 8 and 24, whereas MTX patients showed significant $(P<0.05)$ difference in $\mathrm{C} 1 \mathrm{M}$ levels at week 8 (23\% decrease vs $2 \%$ increase) (Fig. 3a). There was a clear difference in profiles of C1M MTX patients once correlated to DAS28 at week 24, albeit statistically insignificant. Treatment with either TCZ or MTX did not result in significant differences in $\mathrm{C} 2 \mathrm{M}$ profiles of responding and non-responding patients at weeks 8 and 24 (not significant (ns)) (Fig. 3b). MTX showed a trend to increase the C2M level, whereas the level was unchanged with TCZ. C3M profiles did not display any significant differences after treatment with TCZ between responders and non-responders at week 8 and 24; however, MTX patients showed significant difference $(P<0.01)$ in the decrease of C3M levels at week $8(18 \%$ in responders vs $9 \%$ in nonresponders) (Fig. 3c). The statistical significance between MTX C3M profiles increased $(P<0.001)$ once correlated with DAS28 at week 24 (19\% decrease in responders vs $4 \%$ in non-responders). Similarly, treatment with TCZ did not result in significantly different $\mathrm{C} 4 \mathrm{M}$ profiles in responders and non-responders at both time points, while MTX patients displayed significant difference $(P<0.01)$ in the decrease of C4M levels at week 8 (23\% in responders and $9 \%$ in non-responders) with increased significance $(P<0.001)$ once correlated with DAS28 at week 24 ( $21 \%$ in responders and $4 \%$ in non-responders) (Fig. 3d).

Treatment with TCZ did not result in significantly different CRPM profiles among responders and nonresponders at weeks 8 and 24, but MTX patients displayed significantly different $(P<0.05)$ CRPM profiles at week 8 (14\% decrease in responders vs $2 \%$ increase in non-responders) (Fig. 4a). Intriguingly, the opposite profiles of CRPM was observed once correlated with DAS28 at week 24,7\% increase in responders and $3 \%$ decrease in non-responders in CRPM levels $(P<0.05)$.

Table 3 Treatment-dependent difference in core outcome measures used to assess disease activity in rheumatoid arthritis (RA)

\begin{tabular}{|c|c|c|c|c|}
\hline \multirow{3}{*}{$\begin{array}{l}\text { Parameter, \% change } \\
\text { from baseline to week } 8\end{array}$} & \multicolumn{4}{|l|}{ ACR50 Patients } \\
\hline & \multicolumn{2}{|l|}{ Tocilizumab } & \multicolumn{2}{|l|}{ Methotrexate } \\
\hline & Responders & Non-Responders & Responders & Non-Responders \\
\hline DAS28\% mean (Cl-95\%) & $-54.7(-60.1$ to -49.3$)$ & $-30.0(-33.0$ to -27.0$)$ & $-46.4(-52.1$ to -40.7$)$ & $-12.8(-15.7$ to -10.1$)$ \\
\hline SJC, \% mean (Cl-95\%) & $-78.4(-83.8$ to -73.0$)$ & $-29.7(-37.6$ to -21.7$)$ & $-80.7(-89.0$ to -72.5$)$ & $-22.8(-32.1$ to -13.6$)$ \\
\hline TJC, \% mean (Cl-95\%) & $-76.3(-80.7$ to -72.0$)$ & $-25.8(-34.1$ to -17.5$)$ & $-73.6(-81.4$ to -65.8$)$ & $-22.1(-30.1$ to -14.2$)$ \\
\hline VAS Pain, \% mean (Cl-95\%) & $-7.7(-35.2$ to 19.9$)$ & $-10.5(-28.5$ to 7.5$)$ & $-40.8(-68.3$ to -13.4$)$ & $-22.8(-36.6$ to -8.9$)$ \\
\hline
\end{tabular}

Table represent mean percentage change from baseline to week 8 for each respective parameter. DAS28 28-Joint Disease Activity Score, TJC tender joint count, SJC swollen joint count, VAS Pain visual analogue scale pain 


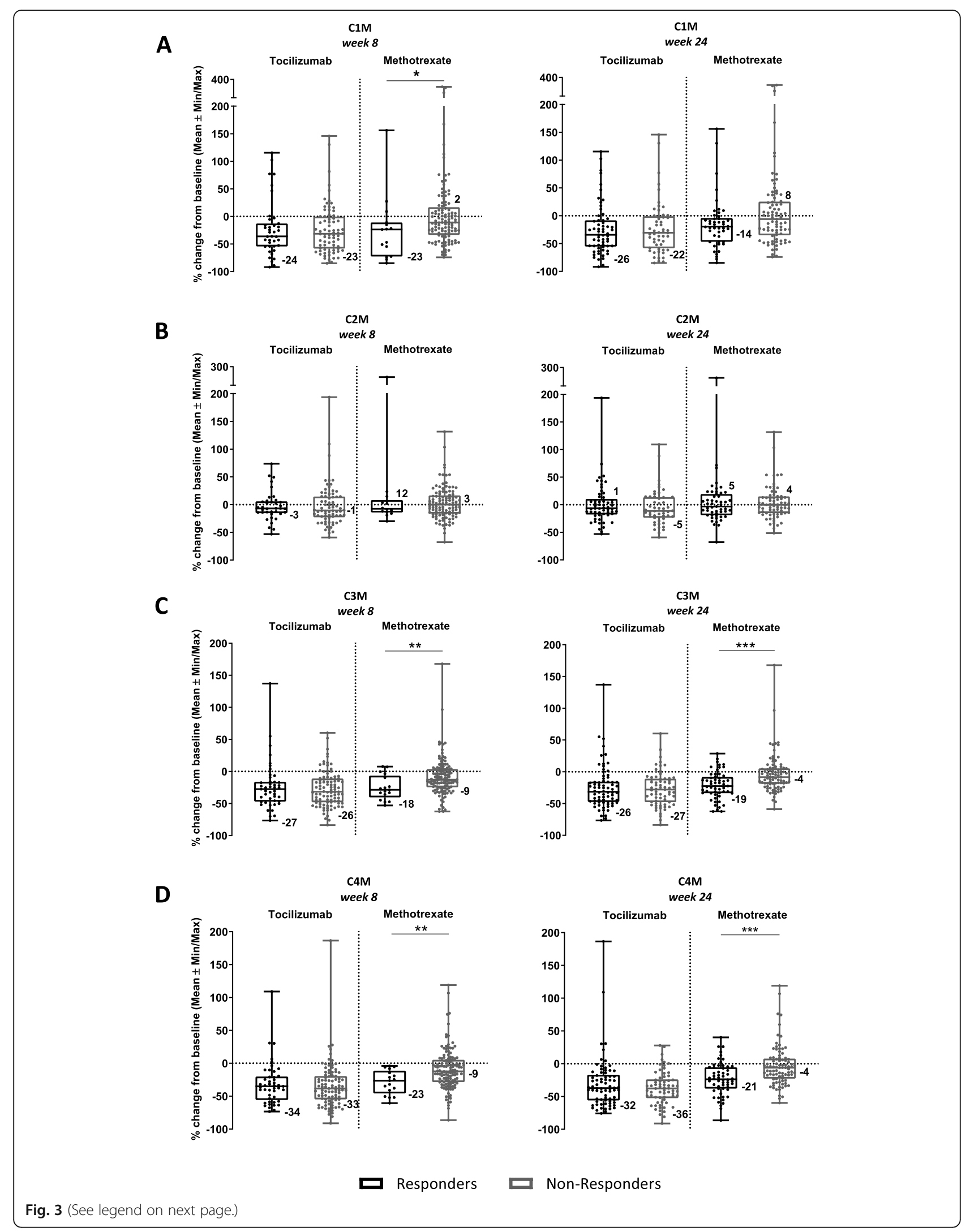


(See figure on previous page.)

Fig. 3 Treatment-dependent difference in degradation biomarker profiles of patients assigned as ACR50 responders (black bars) and ACR50 nonresponders (grey bars) from baseline to week 8 and predictive profiles at week 24. Figures represent serum levels of C1M (a) (TCZ $n=115 ;$ MTX $n=128), C 2 M(\mathbf{b})(T C Z n=109 ;$ MTX $n=122), C 3 M(\mathbf{c})(T C Z n=141 ;$ MTX $n=145)$ and C4M (d) (TCZ $n=140 ;$ MTX $n=146)$ in response to TCZ and MTX at week 8 and predictive profiles at week 24. Data are shown as mean with SEMs

There was observed a strong modulation of the CRP levels among both treatment groups. Patients treated with TCZ displayed a significantly different $(P<0.05)$ decrease of CRP profiles between responders and non-responders at week 8 (91\% vs 59\%) and after correlation to DAS28 at week 24 (76\% vs 54\%) (Fig. 4b). Similarly, treatment with MTX resulted in significantly different $(P<0.01)$ CRP profiles between responders and non-responders at week 8 (56\% decrease vs $9 \%$ increase) with increased

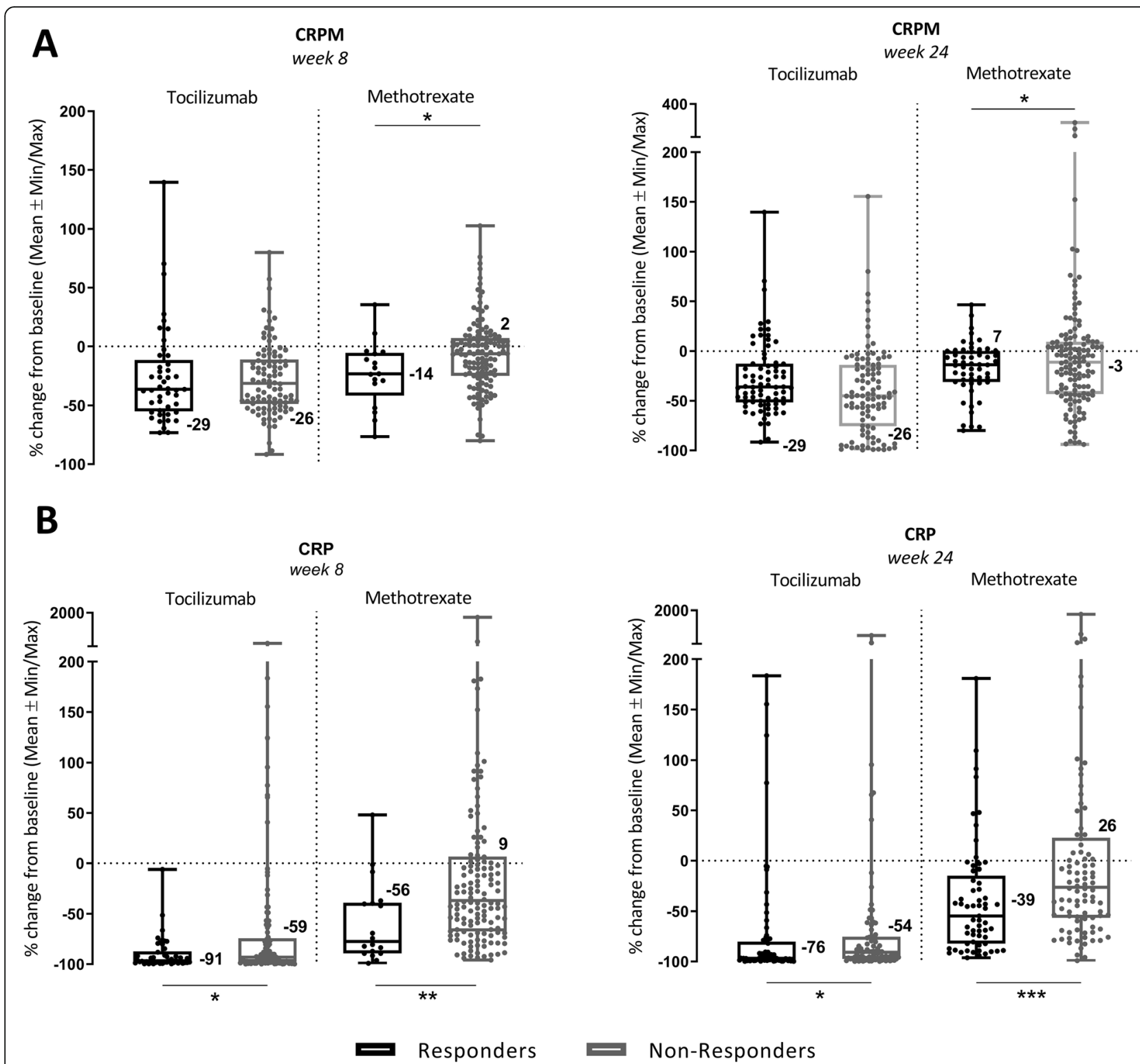

Fig. 4 Treatment-dependent difference in inflammatory biomarker profiles of patients assigned as ACR50 responders (black bars) and ACR50 nonresponders (grey bars) from baseline to week 8 and predictive profiles at week 24. Figures represent serum levels of CRPM (a) (TCZ $n=143 ;$ MTX $n=145)$ and CRP (b) (TCZ $n=164 ;$ MTX $n=161)$ in response to TCZ and MTX at week 8 and predictive profiles at week 24. Data are shown as mean with SEMs 
significance $(P<0.001)$ after correlation to DAS28 at week 24 (39\% decrease vs $26 \%$ increase; Table 3 ).

\section{Correlation between change in biomarker levels and change in disease activity at weeks 8,16 and 24}

To investigate whether differences in tissue degradation and tissue specific inflammation levels can be associated with overall improvement in disease activity, the difference in biomarker levels were correlated to the change in disease activity score (DAS28) at weeks 8, 16 and 24 . The prognostic value of measured biomarkers was assessed by Spearman's rank correlation coefficient (rho) and $P$ value between change in tissue remodelling biomarkers from baseline to week 8 and change in disease activity (DAS28) at week 16 and 24 (Table 4). Correlation values account for Bonferroni correction in order to avoid type I error rate per biomarker. Significance has been adjusted for number of tests performed for each respective biomarker. In this case, significance of $P<0.05$ was adjusted to $P<0.0083$. As a validation step, biomarker levels at baseline in the TCZ group were correlated to DAS28 change at week 16 . These findings were validated by investigating correlation between week 8 biomarker levels with DAS28 change at week 24 in the PBO group. None of the investigated biomarker correlated to DAS28 in either TCZ or PBO group and no significant differences in correlation between both groups were observed (data not shown). In patients treated with tocilizumab (TCZ), only CRP was significantly correlated with change in DAS28 at week $8($ rho $=0.32, P<0.0001)$ and week $16($ rho $=0.31, P=0.0001)$ (Table 4). In patients treated with methotrexate (MTX), three biomarkers correlated with change in DAS28 at all time points (C3M, C4M, CRP). Change in C3M levels correlated to change in
DAS28 at week 8 with correlation coefficient of $0.32(P=$ $0.0001)$ at week $8,0.3(P=0.0005)$ at week 16 and 0.26 $(P=0.003)$ at week 24. Change in levels of C4M correlated with change in DAS28 with correlation coefficient of 0.35 $(P<0.0001)$ at week $8,0.32(P=0.0001)$ at week 16 and $0.26(P=0.002)$ at week 24 . Change in levels of CRP correlated with change in DAS28 with correlation coefficient of $0.41(P<0.0001)$ at week $8,0.34(P<0.0001)$ at week 16 and $0.25(P=0.0026)$ at week 24 . Moreover, change in levels of CRPM correlated significantly with change in DAS28 with correlation coefficient of $0.29(P=0.0006)$ at week 8 .

\section{Discussion}

In this sub-study of the AMBITION trial, we investigated association of different tissue remodelling biomarkers with aspects of joint degradation and inflammation in RA. We investigated the biomarker profiles in response to TCZ, MTX and placebo. In the full AMBITION study, clinical signs and symptoms after treatment with TCZ and MTX have been examined, but tissue remodelling biomarkers have not been investigated [27]. The approach involving tissue remodelling biomarker assessment gives a unique insight into the drug's mode of action at the tissue level and ongoing tissue remodelling processes.

Results of this study confirmed that investigated biomarkers are significantly upregulated in rheumatoid arthritis (Table 2), suggesting that an ongoing excessive ECM remodelling results in the excessive release of these biomarkers from the affected joint structures to circulation.

We demonstrated that there was significant difference between the two treatments and placebo patients in

Table 4 Correlation between week 8 change in levels of respective biomarkers according to the treatment group and change in disease activity (DDAS28) at weeks 8, 16 and 24. Correlation values are given as Spearman's correlation coefficient (rho) and significance as $P$ value. Significant correlations with $P<0.0083$ have been highlighted (Bonferroni correction)

\begin{tabular}{|c|c|c|c|c|c|c|}
\hline \multirow[t]{2}{*}{ Biomarker } & \multicolumn{3}{|l|}{ Tocilizumab } & \multicolumn{3}{|l|}{ Methotrexate } \\
\hline & $\triangle \mathrm{DAS28}$ week 8 & $\Delta$ DAS28 week 16 & $\triangle$ DAS28 week 24 & $\triangle \mathrm{DAS28}$ week 8 & $\Delta$ DAS28 week 16 & $\triangle \mathrm{DAS28week} 24$ \\
\hline \multirow[t]{2}{*}{ C1M } & rho $=0.08$ & rho $=0.06$ & rho $=0.002$ & rho $=0.11$ & rho $=0.06$ & rho $=0.08$ \\
\hline & $P=0.43$ & $P=0.53$ & $P=0.98$ & $P=0.22$ & $P=0.53$ & $P=0.4$ \\
\hline \multirow[t]{2}{*}{$\mathrm{C} 2 \mathrm{M}$} & rho $=0.02$ & rho $=0.03$ & rho $=0.02$ & rho $=0.04$ & rho $=-0.04$ & rho $=0.02$ \\
\hline & $P=0.87$ & $P=0.73$ & $P=0.83$ & $P=0.66$ & $P=0.69$ & $P=0.87$ \\
\hline \multirow[t]{2}{*}{ C3M } & rho $=0.1$ & rho $=0.12$ & rho $=0.05$ & rho $=0.32$ & rho $=0.3$ & rho $=0.26$ \\
\hline & $P=0.24$ & $P=0.16$ & $P=0.54$ & $P=0.0001$ & $P=0.0005$ & $P=0.003$ \\
\hline \multirow[t]{2}{*}{ C4M } & rho $=0.08$ & rho $=0.08$ & rho $=-0.01$ & rho $=0.35$ & rho $=0.32$ & rho $=0.26$ \\
\hline & $P=0.38$ & $P=0.37$ & $P=0.89$ & $P<0.0001$ & $P=0.0001$ & $P=0.002$ \\
\hline \multirow[t]{2}{*}{ CRP } & rho $=0.32$ & rho $=0.31$ & rho $=0.21$ & rho $=0.41$ & rho $=0.34$ & rho $=0.25$ \\
\hline & $P<0.0001$ & $P=0.0001$ & $P=0.01$ & $P<0.0001$ & $P<0.0001$ & $P=0.0026$ \\
\hline \multirow[t]{2}{*}{ CRPM } & $\mathrm{rho}=0.13$ & rho $=0.13$ & rho $=0.1$ & rho $=0.29$ & rho $=0.18$ & rho $=0.21$ \\
\hline & $P=0.13$ & $P=0.14$ & $P=0.24$ & $P=0.0006$ & $P=0.039$ & $P=0.016$ \\
\hline
\end{tabular}


modulation of tissue remodelling biomarkers. Levels of $\mathrm{C} 1 \mathrm{M}, \mathrm{C} 3 \mathrm{M}$ and $\mathrm{C} 4 \mathrm{M}$ were significantly suppressed by TCZ compared to MTX, suggesting that TCZ exhibit more tissue protective effect towards different joint structures than that of MTX. TCZ had a minimal and MTX no effect on cartilage degradation measured by C2M. We observed a great inhibition of CRP and significant inhibition of CRPM after treatment with TCZ, whereas MTX had limited effect on their levels. This can be explained by the fact that IL- 6 receptor is a main target for TCZ treatment and CRP is produced in the liver in the response to high levels of IL-6. Thus, TCZ should suppress CRP, which is observed and further downstream suppression of CRPM, which is observed as expected. The superior effect of TCZ in controlling of CRP and CRPM levels in the present study can be compared with LITHE study where TCZ inhibited completely the level of CRP and in the large extent level of CRPM [20]. These results indicate that TCZ is more effective than MTX treatment that better limits tissue turnover and inflammation. More importantly, it indicates that measured biomarkers can be successfully applied to reveal differences in the mode of action between the investigated treatments.

We examined biomarker profiles of ACR50 responding and non-responding patients to investigate if any of the biomarker could stratify patients that benefited from treatments already at week 8 . The significant differences in tissue remodelling biomarker profiles between treatment responders and non-responders were observed primarily among patients treated with MTX. This was an interesting observation considering that MTX patients had less biomarker downmodulation than TCZ patients. Despite the fact that TCZ patients were more likely to reach ACR50 than MTX patients, the significant differences in biomarker profiles discriminating responders from non-responders were observable only for CRP. Previously, similar studies have shown that combination treatment involving TCZ+MTX do not necessarily display differences in profiles of tissue turnover biomarkers between ACR50 responders and non-responders already at week 8 [20]. These differences were observable at weeks 16 and 24, which could explain results seen in TCZ patients. Interestingly, results of this study showed that the separation of these treatments allowed to observe differences in biomarker profiles of patients treated with MTX monotherapy as early as at week 8 . This indicates that the neo-epitope biomarkers can be potentially used as a tool to identify treatment responding and non-responding patients at the early treatment time point. As a possible explanation behind the observed differences in biomarker profiles might be different mechanisms underlying the mode of action of TCZ and MTX. TCZ limits the inflammation by selective inhibition of IL-6 receptors and forming the receptor-complex that prevents IL-6 signal transduction that stimulates $\mathrm{B}$ and $\mathrm{T}$ cells. MTX as opposed to TCZ is a broad spectrum, systemic immune system suppressant acting on a wide range of immune cells and cytokines production. As a consequence of these treatment-dependent differences, joint structures might be potentially differently modulated and result in distinct biomarker profiles between treatments.

Biomarkers of tissue turnover have shown a great prognostic potential and can predict long-term clinical response to treatment already at the early time point [26]. It is of great importance to determine at the possibly earliest stage whether patient will respond to the treatment in the future and benefit from the treatment. We demonstrated that only change to week 8 in the CRP levels significantly predicted clinical response to TCZ and correlated with the change in the disease activity at weeks 16 and 24 as a response to treatment (Table 4). Interestingly, more biomarkers were predictive for MTX treatment. In this case, change to week 8 in the C3M, C4M, CRP and CRPM levels significantly predicted clinical response at weeks 16 and 24.

These results show a great value of the biomarkers which can be a determining indicator of patient clinical benefit from the given treatment. Previous studies have shown that biomarker levels reflecting structural joint damage such as $\mathrm{C} 1 \mathrm{M}, \mathrm{C} 2 \mathrm{M}$ and $\mathrm{C} 3 \mathrm{M}$ can be associated with disease progression and disease activity over time [22, 32].

Our results confirm similar biomarker analysis performed for LITHE study that measured C2M, C3M, CRPM and CRP, but in patients treated with combination therapy involving TCZ at two doses (8 and $4 \mathrm{mg}$ / $\mathrm{kg}$ ) with MTX. The combination therapy of TCZ and MTX decreased C3M and CRPM levels at week 8 in a comparable extent to TCZ alone in our study [20]. There was a greater decrease in levels of $C 2 M$ and CRP at week 8 after TCZ+MTX treatment, albeit TCZ monotherapy significantly decreased levels of CRP proving its positive effect on inflammation process. We observed a limited effect of TCZ alone on cartilage balance measured with $\mathrm{C} 2 \mathrm{M}$; however, high $\mathrm{C} 2 \mathrm{M}$ upregulation is typical at erosive disease stages and that was not a case in the examined cohort. Another study involving the LITHE cohort investigated the effect of structural joint damage and progression, measured with C1M [22]. TCZ in combination with MTX decreased levels of C1M at week 8 in a greater extent than TCZ monotherapy, but both treatments provided significant downmodulation of $\mathrm{C} 1 \mathrm{M}$ and protective effect on connective tissues. Gudmann et al. investigated the baseline membrane remodelling measured with $\mathrm{C} 4 \mathrm{M}$ after $\mathrm{TCZ}+\mathrm{MTX}$ treatment in LITHE and RADIATE cohorts [23]. Reported results 
support our findings, where TCZ+MTX treatment downregulated levels of $\mathrm{C} 4 \mathrm{M}$ at week 8 in the similar extent as TCZ monotherapy, proving that both treatment strategies have protective effect on tissue remodelling. These results indicate that TCZ monotherapy can be successfully applied as an effective alternative to commonly used combined therapy involving TCZ+MTX. One of the main limitations of this study was that we did not measure biomarkers at other time points than baseline and week 8 , so we could not anticipate the long-term treatment effect on biomarker modulation. Another limitation of this study was no radiographic to support our findings of tissue remodelling. However, radiographic showed sensitivity limitations in detecting early structural changes in joints and surrounding structures, indicating their limited applicability in this type of study [33].

\section{Conclusion}

Patients receiving TCZ treatment for 8 weeks have clearly higher suppression of tissue remodelling and inflammatory biomarkers over patients treated with MTX. The decrease of the inflammatory process is believed to be associated with decrease of ECM remodelling and tissue degradation, which is supported by this study. On the other hand, patients receiving MTX enable for a clear discrimination of biomarker profiles and identification of those who were most likely to benefit from the treatment at the early time point. The results of this study highlight that biomarkers reflecting tissue turnover can be applied as disease and response to therapy markers. Their application may facilitate future identification of patients most likely to benefit from a given treatment with the aim to develop possibly best personalised treatment strategy.

\section{Abbreviations \\ ACR50: 50\% disease improvement; BMI: Body mass index; C1M: Type I collagen degradation biomarker; C2M: Type II collagen degradation biomarker; C3M: Type III collagen degradation biomarker; C4M: Type IV collagen degradation biomarker; CRP: C-reactive protein; CRPM: C-reactive protein degradation biomarker; $\mathrm{CV}$ : Coefficient of variance; DAS28: Disease Activity Score in 28 joints; DMARDs: Disease-modifying anti-rheumatic drugs; ECM: Extracellular matrix; IL-6: Interleukin-6; LLOD: Lower limit of detection; MMP: Matrix metalloproteinase; MTX: Methotrexate; PBO: Placebo; RA: Rheumatoid arthritis; TCZ: Tocilizumab; TMB: 3,3',5,5'- \\ Tetramethylbenzidine substrate; ULOD: Upper limit of detection}

\section{Acknowledgements}

We would also like to thank all the patients who donated their blood for the purposes of this study.

\section{Authors' contributions}

All authors were involved with the design of the study, interpretation of data, critical revising of the manuscript and approving the final version for submission. ACBJ, ASO and MK were primarily responsible for the data acquisition, whereas PAD and SSA primarily did the analysis of the data and drafted the manuscript. PAD (pad@nordicbio.com) and ASO (aso@nordicbio. com) take full responsibility of the integrity of the work from inception to finished article.

\section{Funding}

This work was supported by the University of Copenhagen (KU) and Nordic Bioscience. The KU was not involved in the study design, collection, analysis and interpretation of data, writing or deciding to submit the manuscript for publication. Nordic Bioscience was involved in the study design, collection, analysis, interpretation of data, writing the manuscript and the decision to submit the manuscript for publication.

\section{Availability of data and materials}

The datasets used and/or analysed during the current study are available from the corresponding author on reasonable request.

\section{Ethics approval and consent to participate}

The AMBITION study (NCT00109408) was approved by the ethics committee at each participating institution (in USA, Canada and Israel) and was conducted in accordance with the principles of good clinical practice and according to the Declaration of Helsinki. All patients included provided written, informed consent before inclusion in the study.

\section{Consent for publication}

Not applicable.

\section{Competing interests}

Anne C. Bay-Jensen (ACBJ), Morten Karsdal (MK), Anne Siebuhr (ASO) and Samra Sardar (SSA) are full-time employees and/or shareholders at Nordic Bioscience. Patryk Drobinski (PAD) is a full-time employee at University of Copenhagen. PAD has no disclosures and no competing interests. None of the authors received any kind of financial benefits or other bonuses for the work described in this manuscript.

\section{Author details}

${ }^{1}$ ImmunoScience, Nordic Bioscience, Herlev Hovedgade 207, DK-2730 Herlev, Denmark. ${ }^{2}$ Department of Biomedical Sciences, University of Copenhagen, Blegdamsvej 3, 2200 Copenhagen N, Denmark. ${ }^{3}$ Biomarkers and Research, Nordic Bioscience, Herlev Hovedgade 207, DK-2730 Herlev, Denmark.

Received: 29 June 2020 Accepted: 24 November 2020

Published online: 07 January 2021

\section{References}

1. Dougados M. Comorbidities in rheumatoid arthritis. Curr Opin Rheumatol. 2016;28(3):282-8 https://doi.org/10.1097/BOR.0000000000000267.

2. Mcinnes IB, Schett G. The pathogenesis of rheumatoid arthritis. N Engl J Med. 2011:365:2205-19 https://doi.org/10.1056/NEJMra1004965.

3. Klareskog L, Padyukov L, Lorentzen J, Alfredsson L. Mechanisms of disease: genetic susceptibility and environmental triggers in the development of rheumatoid arthritis. Nature. 2006;2(8):425-33 https://doi.org/10.1038/ ncprheum0249.

4. Sardar S, Andersson $\AA$. Old and new therapeutics for rheumatoid arthritis: in vivo models and drug development. Immunopharmacol Immunotoxicol. 2016:1-12 https://doi.org/10.3109/08923973.2015.1125917.

5. Gaffo A, Saag K, Curtis J. Treatment of rheumatoid arthritis. Am J Health-Syst Pharm. 2006;63:2451-65 https://doi.org/10.2146/ajhp050514.

6. Reynolds A, Koening AS, Bananis E, Singh A. When is switching warranted among biologic therapies in rheumatoid arthritis? Expert Rev Pharmacoecon Outcomes Res. 2012;12(3):319-33 https://doi.org/10.1586/ erp.12.27.

7. Yoshizaki K, Nishimoto N, Mihara M, Kishimoto T. Therapy of rheumatoid arthritis by blocking IL-6 signal transduction with a humanized anti-IL-6 receptor antibody. Springer Semin Immunopathol. 1998;20:247-59 https:// doi.org/10.1007/bf00832010.

8. Karsdal MA, Schett G, Emery P, et al. IL-6 receptor inhibition positively modulates bone balance in rheumatoid arthritis patients with an inadequate response to anti-tumor necrosis factor therapy: biochemical marker analysis of bone metabolism in the Tocilizumab RADIATE study. Semin Arthritis Rheum. 2012;42(2):131-9 https://doi.org/10.1016/j.semarthrit. 2012.01.004

9. Finzel $\mathrm{S}$, Kraus $\mathrm{S}$, Figueiredo $\mathrm{CP}$, et al. Comparison of the effects of tocilizumab monotherapy and adalimumab in combination with methotrexate on bone erosion repair in rheumatoid arthritis. Ann Rheum Dis. 2019;6:1186-91 https://doi.org/10.1136/annrheumdis-2018-214894. 
10. Sack U, Kinne R, Marx T, Heppt P, Bender S, Emmrich F. Interleukin-6 in synovial fluid is closely associated with chronic synovitis in rheumatoid arthritis. Rheumatol Int. 1993;13:45-51 https://doi.org/10.1007/BF00307733.

11. Madhok R, Crilly A, Watson J, Capell HA, Royal G. Serum interleukin 6 levels in rheumatoid arthritis: correlations with clinical and laboratory indices of disease activity. Ann Rheum Dis. 1993;52:232-4 https://doi.org/10.1136/ard. 52.3.232.

12. Kremer JM, Blanco R, Brzosko M, et al. Tocilizumab inhibits structural joint damage in rheumatoid arthritis patients with inadequate responses to methotrexate: results from the double-blind treatment phase of a randomized placebo-controlled trial of tocilizumab safety and prevention of structural joint damage at one year. Arthritis Rheum. 2011;63(3):609-21 https://doi.org/10.1002/art.30158

13. Mcinnes IB, Schett G. Cytokines in the pathogenesis of rheumatoid arthrits. Nat Rev Immunol. 2007;7:429-42 https://doi.org/10.1038/nri2094.

14. Karsdal MA, Nielsen MJ, Sand JM, et al. Extracellular matrix remodeling: the common denominator in connective tissue diseases. Assay Drug Dev Technol. 2013;11(2):70-92 https://doi.org/10.1089/adt.2012.474.

15. Lu P, Takai K, Weaver VM, Werb Z. Extracellular matrix degradation and remodeling in development and disease. Cold Spring Harb Perspect Biol. 2011;3 https://doi.org/10.1101/cshperspect.a005058.

16. Mohammed FF, Smookler DS, Khokha R. Metalloproteinases, inflammation, and rheumatoid arthritis. Ann Rheum Dis. 2003;62:43-7 https://doi.org/10. 1136/ard.62.suppl_2.ii43.

17. Burrage PS, Mix KS, Brinckerhoff CE. Matrix metalloproteinases: role in arthritis. Front Biosci. 2006;11:529-43 https://doi.org/10.2741/1817.

18. Siebuhr AS, Sun S, Byrjalsen I. Suppression of active, but not total MMP-3, is associated with treatment response in a phase III clinical study of rheumatoid arthritis. Clin Exp Rheumatol. 2018;36:94-101 https://www. clinexprheumatol.org/abstract.asp?a=11449.

19. Karsdal MA, Henriksen K, Leeming DJ, et al. Biochemical markers and the FDA critical path: how biomarkers may contribute to the understanding of pathophysiology and provide unique and necessary tools for drug development. Biomarkers. 2009;14(3):181-202 https://doi.org/10.1080/ 13547500902777608

20. Bay-Jensen AC, Platt A, Byrjalsen I, Vergnoud P, Christiansen C, Karsdal MA. Effect of tocilizumab combined with methotrexate on circulating biomarkers of synovium, cartilage, and bone in the LITHE study. Semin Arthritis Rheum. 2014;43(4):470-8 https://doi.org/10.1016/j.semarthrit.2013. 07.008.

21. Bay-Jensen AC, Platt A, Siebuhr AS, Christiansen C, Byrjalsen I, Karsdal MA. Early changes in blood-based joint tissue destruction biomarkers are predictive of response to tocilizumab in the LITHE study. Arthritis Res Ther. 2016:7-15 https://doi.org/10.1186/s13075-015-0913-x.

22. Siebuhr AS, Bay-Jeensen AC, Leeming DJ, et al. Serological identification of fast progressors of structural damage with rheumatoid arthritis. Arthritis Res Ther. 2013;15:1-9 https://doi.org/10.1186/ar4266.

23. Gudmann NS, Junker $P$, Juhl $P$, et al. Type IV collagen metabolism is associated with disease activity, radiographic progression and response to tocilizumab in rheumatoid arthritis. Clin Exp Rheumatol. 2018;36:829-35 https://www.clinexprheumatol.org/article.asp?a=12293.

24. Kojima T, Yabe Y, Kaneko A, Hirano Y, Ishikawa H, Hayashi M. Monitoring C-reactive protein levels to predict favourable clinical outcomes from tocilizumab treatment in patients with rheumatoid arthritis. Mod Rheumatol. 2013;23:977-85 https://doi.org/10.1007/s10165012-0782-y.

25. Juhl P, Thudium CS, Gudmann NS, Karsdal MA, Siebuhr AS. IL-6 receptor inhibition modulates type III collagen and C-reactive protein degradation in rheumatoid arthritis patients with an inadequate response to anti-tumour necrosis factor therapy: analysis of connective tissue turnover in the tocilizumab RADIATE study. Clin Exp Rheumatol. 2018;36:568-74 https://www.clinexprheumatol.org/article. asp?a $=12085$

26. Bay-jensen A, Byrjalsen I, Siebuhr AS, et al. Serological biomarkers of joint tissue turnover predict tocilizumab response at baseline. J Clin Rheumatol. 2014;20(6):332-5 https://doi.org/10.1097/RHU. 0000000000000150 .

27. Jones G, Sebba A, Gu J, et al. Comparison of tocilizumab monotherapy versus methotrexate monotherapy in patients with moderate to severe rheumatoid arthritis: the AMBITION study. Ann Rheum Dis. 2010;69:88-96 https://doi.org/10.1136/ard.2008.105197.
28. Leeming DJ, He Y, Veidal SS, et al. A novel marker for assessment of liver matrix remodeling: an enzyme-linked immunosorbent assay (ELISA) detecting a MMP generated type I collagen neo-epitope (C1M). Biomarkers. 2011;16(7) https://doi.org/10.3109/1354750X.2011.620628.

29. Bay-Jensen AC, Liu Q, Byrjalsen I, et al. Enzyme-linked immunosorbent assay (ELISAs) for metalloproteinase derived type II collagen neoepitope, CIIM increased serum CIIM in subjects with severe radiographic osteoarthritis. Clin Biochem. 2011;44:423-9 https://doi.org/10.1016/j.clinbiochem.2011.01. 001.

30. Barascuk N, Veidal SS, Larsen L, Larsen DV, Larsen MR, et al. A novel assay for extracellular matrix remodeling associated with liver fibrosis: an enzymelinked immunosorbent assay (ELISA) for a MMP-9 proteolytically revealed neo-epitope of type III collagen. Clin Biochem. 2010;43:899-904 https://doi. org/10.1016/j.clinbiochem.2010.03.012.

31. Karsdal MA, Genovese F, Madsen EA, Manon-Jensen T, Schuppan D. Collagen and tissue turnover as function of age: implications for fibrosis. J Hepatol. 2015;64(1) https://doi.org/10.1016/j.jhep.2015.08. 014.

32. Bay-Jensen AC, Wichuk S, Byrjalsen I, et al. Circulating protein fragments of cartilage and connective tissue degradation are diagnostic and prognostic markers of rheumatoid arthritis and ankylosing spondylitis. PLoS One. 2013; 8(1):1-7 https://doi.org/10.1371/journal.pone.0054504.

33. Sokka T. Radiographic scoring in rheumatoid arthritis: a short introduction to the methods. Bull NYU Hosp Jt Dis. 2008;66(2):166-8 https://pubmed. ncbi.nlm.nih.gov/18537790/.

\section{Publisher's Note}

Springer Nature remains neutral with regard to jurisdictional claims in published maps and institutional affiliations.
Ready to submit your research? Choose BMC and benefit from:

- fast, convenient online submission

- thorough peer review by experienced researchers in your field

- rapid publication on acceptance

- support for research data, including large and complex data types

- gold Open Access which fosters wider collaboration and increased citations

- maximum visibility for your research: over $100 \mathrm{M}$ website views per year

At BMC, research is always in progress.

Learn more biomedcentral.com/submissions 